\title{
Analysis of current conversion primary sensors dynamic characteristics of a reactive power source with renewable energy sources into secondary voltage
}

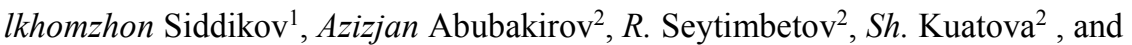 \\ Yuliya Lezhnina ${ }^{3 *}$ \\ ${ }^{1}$ Tashkent University of Information Technologies named after Muhammad al-Kharazmi, 100200 \\ Tashkent, Uzbekistan \\ ${ }^{2}$ Karakalpak State University named after Berdaq 742012, Nukus, Uzbekistan \\ ${ }^{3}$ Astrakhan State University of Architecture and Civil Engineering, 414056, Astrakhan, Russia
}

\begin{abstract}
This paper presents the structure of a sensor for converting multiphase primary currents into a secondary voltage of reactive power networks with renewable energy sources, an algorithm for modeling processes in the sensor, which provides control over the primary current and a control device. The dynamic characteristics of the investigated sensor are given on the basis of the proposed analytical expressions. A graph model to study the conversion processes into secondary output voltages has been created. The sensor has been shown to be capable of generating a secondary signal at high speed.
\end{abstract}

\section{Introduction}

Much attention is paid in the world to the improvement of control and management elements, devices that determine strictly established operation modes of primary energy sources of power supply systems (SS), which provide fixed values and parameters in various energy conversion systems. For control and management of power supply systems using renewable energy sources (RES) in developed countries, the development of primary current sensors models for the values and parameters of electrical energy reactive power is considered relevant. Therefore, the development, research and implementation of the principles of their construction are important in this direction. In this regard, it is necessary to develop models, algorithms and software solutions for the elements and devices that ensure reliable operating modes of power supply systems, the amount and quality of generated electricity, as well as control and management of the reactive power sources primary currents.

Dynamic characteristics of renewable energy sources power supply systems' reactive power sources, multiphase current sensors of reactive power sources are one of their main

\footnotetext{
* Corresponding author: lejninau@mail.ru
} 
characteristics, reflecting various values and parameters of input primary currents, magnitudes of external influences, power load parameters and other influences [1-3].

The dynamic characteristics of the sensors are determined analytically. The graphical model describing the dynamic operation of the sensor gives an opportunity to study the entire set of physical processes occurring in it, as well as the sources of reactive power, taking into account the operating conditions of the electrical network [4-8].

The dynamic state of the sensor is determined on the basis of the sensor structural diagram, which connects the output signal with the dimensions and parameters of the input, the characteristics of the transverse, longitudinal and vertically distributed parameters of the switching part. Values and parameters of reactive power supply PS networks currents can radically change the dynamic characteristics with inconsistent and nonlinear changes in the characteristics of the number and phase of input quantities under the operating conditions of the sensors.

\section{Materials and methods}

The study of the sensor dynamic characteristics for converting primary currents into secondary voltages of reactive energy and power coming from networks of renewable energy sources is carried out on the basis of modeling processes in the sensor, a graph model and analytical descriptions of the dynamics [1-2].

General PS RES network structure and directions of primary currents $I_{A \gamma}, I_{B \gamma}, I_{C \gamma}, I_{A \Delta}$, $I_{B \Delta}, I_{C \Delta}$ are the sources of reactive power in it and the principle of installing the sensor in PSS and are shown in Figure 1.

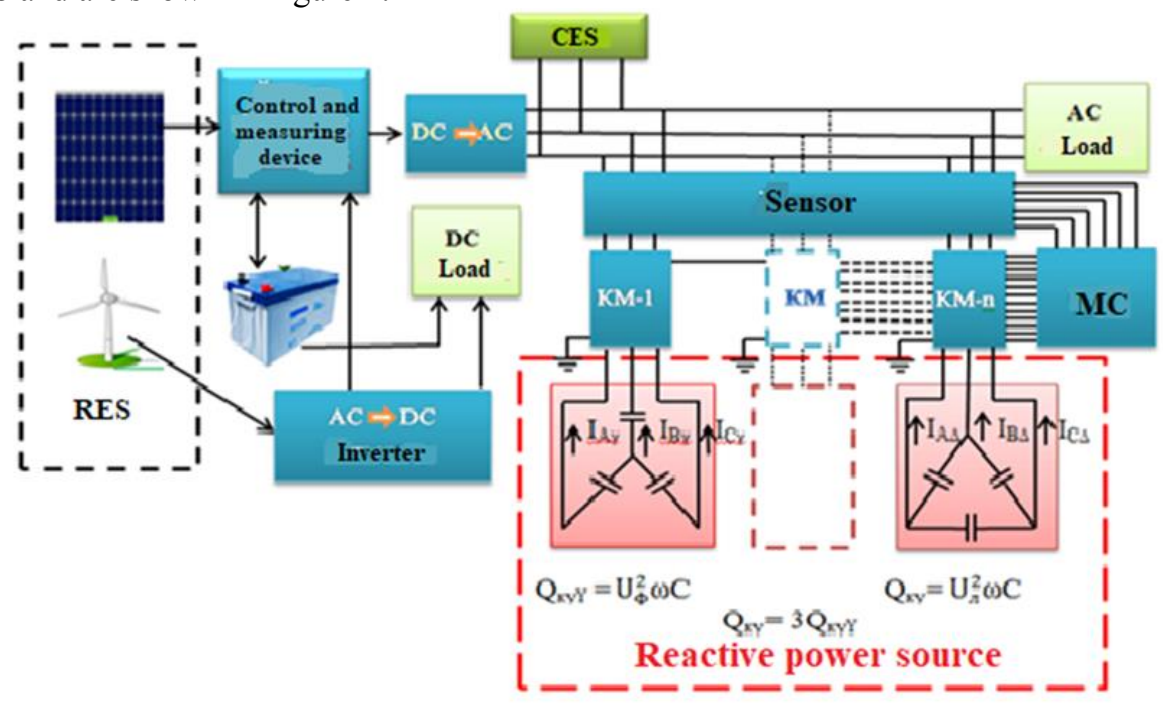

Fig. 1. Connection diagram of sources and consumers PS for RES with the ability to assess energy efficiency using a cloud computing model, install and connect sensors for multiphase primary currents of reactive power.

Here RES is a renewable energy source, CES is a centralized power supply system, DC $\rightarrow \mathrm{AC}-$ is a device that converts alternating current into alternating current; dc load determines the load of dc devices; an alternating current alternating current alternating current converter; Contactors $\mathrm{KM}-1, \mathrm{KM}-\mathrm{n}$; $\mathrm{MC}$ - micro-processor control; $\mathrm{AC}$ is $\mathrm{AC}$ current load. 


\subsection{Signal conversion process}

Investigation of the dynamic characteristics of sensors for converting the primary current of reactive power sources PS into secondary voltage $I_{e}$ defines the sensitive elements Magnetic element currents $\Phi_{\mu}$, generating $\mathrm{U}_{\text {output }}$ - the output voltages of the sensor with primary currents, cross-sectional area, number of turns of the sensor $\mathrm{S}_{\mathrm{CE}}$, WSS is necessary to determine whether the geometric dimensions of the section depend $l_{x . o}$ from permissible ranges of variation and variable geometric dimensions of the magnetic circuit [1-5].

Forms of magnetic transformation sections - the magnetic cores of a multiphase primary current sensor - $I_{A \gamma}, I_{B \gamma}, I_{C \gamma}, I_{A \Delta}, I_{B \Delta}, I_{C \Delta}$ - from reactive power source PS from RES are shown in Fig. 2.

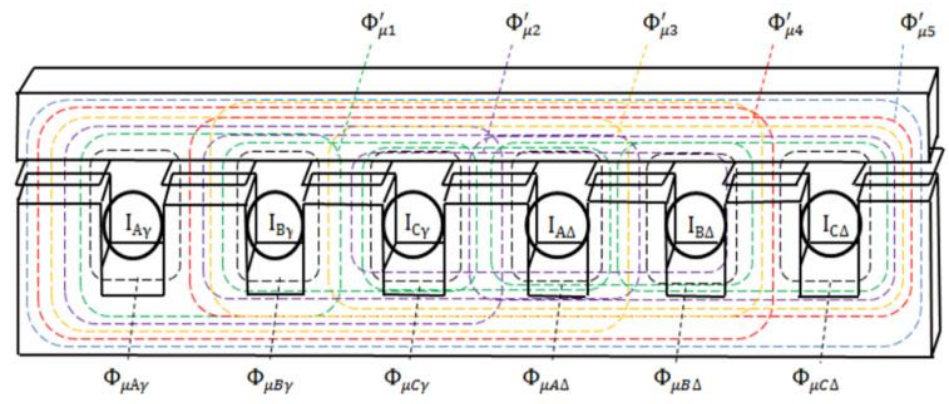

Fig. 2. Magnetic part of the sensor, converting primary currents into secondary voltage

When the primary currents PS of reactive power sources pass first through $I_{A \gamma}$, then through $I_{B \gamma}$, through $I_{C \gamma}, I_{A \Delta}, I_{B \Delta}$ or $I_{C \Delta}$, the coil fields in a common magnetic circuit and parallel to the cores, magnetic currents are generated $\Phi_{\mu \mathrm{A} \gamma}, \Phi_{\mu \mathrm{B} \gamma}, \Phi_{\mu \mathrm{C} \gamma}, \Phi_{\mu \mathrm{A} \Delta}, \Phi_{\mu \mathrm{B} \Delta}$ and $\Phi_{\mu \mathrm{C} \Delta}$, which also pass through the air gap between the cores.

Magnetic PS currents $\Phi_{\mu \mathrm{A} \gamma}, \Phi_{\mu \mathrm{B} \gamma}, \Phi_{\mu \mathrm{C} \gamma}, \Phi_{\mu \mathrm{A} \Delta}, \Phi_{\mu \mathrm{B} \Delta}$ and $\Phi_{\mu \mathrm{C} \Delta}$ of the reactive power supply with a secondary signal for monitoring and control of primary current, single-phase $I_{A \gamma}$, biphasic $I_{A \gamma}, I_{B \gamma}$ or star connection of reactive power sources $I_{B \gamma}, I_{C \gamma}$ and three-phase $I_{A \gamma}$, $I_{B \gamma}, I_{C \gamma}$ and with triangular single-phase connection $I_{A \Delta}$ with two-phase $I_{A \Delta}, I_{B \Delta}$ or $I_{B \Delta}, I_{C \Delta}$ and three-phase $I_{A \Delta}, I_{B \Delta}, I_{C \Delta}$ currents, the sensitive element (at the outputs of conventional or flat measuring coils, balanced magnetic switches, etc.) generates signals in the form of voltage $\mathrm{W}$ - in the quantities corresponding to the reactive power sources' currents.

When monitoring and controlling primary currents generated by power supplies PS and transmitted through the transmission lines using modern electronic and microprocessor means at the rated current of the electrical device or 20 volts at the outputs of the sensitive elements at the nominal cross-sectional area of the conductor for a long time, voltage generation requires $U_{a \gamma}, U_{b \gamma}, U_{c \gamma}, U_{a \Delta}, U_{B \Delta}$, and $U_{c \Delta} . I_{A \gamma}, I_{B \gamma}, I_{C \gamma}, I_{A \Delta}, I_{B \Delta}, I_{C \Delta}$ generation currents, F $\mu$ - magnetic driving forces (m.u.c.), m.y.c., generated by ECT sources and flowing through power lines, $\Phi \mu$ is the magnetic currents cross the surfaces of sensing elements located on a suitable base in the magnetic conversion section and based on interacting magnetic currents, $I_{A \gamma}, I_{B \gamma}, I_{C \gamma}, I_{A \Delta}, I_{B \Delta}, I_{C \Delta}$ - are the primary currents, $U_{a \gamma}, U_{b \gamma}$, $U_{c \gamma}, U_{a \Delta}, U_{6 \Delta}$, and $U_{c \Delta}$ - are the secondary voltage output signals.

\subsection{Research model}

Fig. 3 shows a graph model corresponding to the commutation sensor magnetic part structure, which generates a signal in the form of a secondary voltage for monitoring and controlling the primary currents of reactive power sources PS and the processes occurring in the magnetic part of the commutation [6]. 
In a graph model corresponding to the sensor magnetic part structure and reflecting the processes occurring in the magnetic variable part, $\mathrm{K}_{\mathrm{F} \mu \mathrm{Up}}=\mathrm{w}_{2}-\Phi_{\mu}$ - is the coefficient of the relationship between magnetic fluxes and $U_{\text {output }}$ - is an output voltage, which is measured by the output voltage, which can take the values up to $\mathrm{w}_{2}=1 \div 20$ as needed $[7,9-11]$.

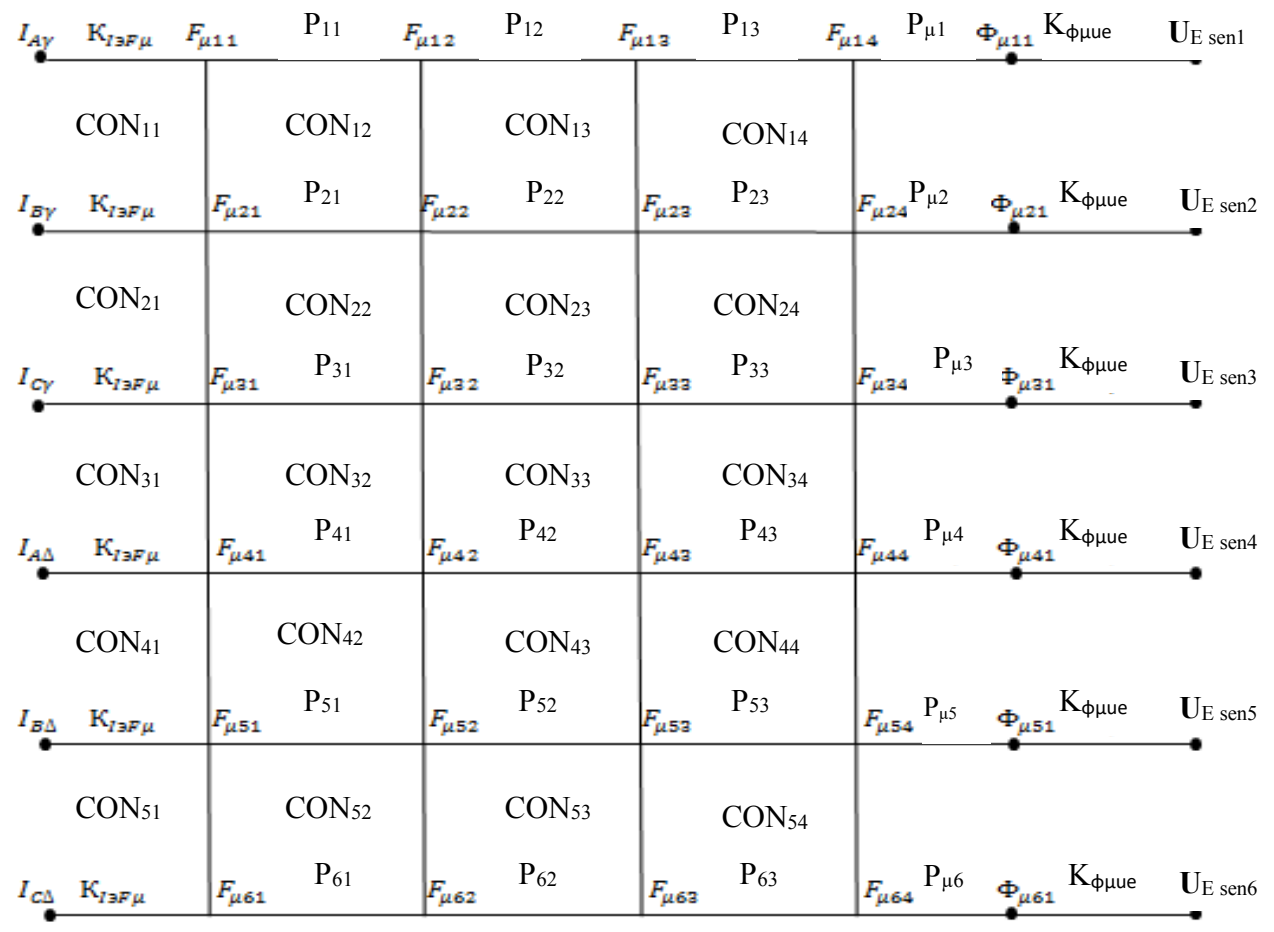

Fig. 3. The process of converting multiphase primary currents to a secondary voltage and a model of the sensor conversion structure

\subsection{Analytical description of dynamics}

Investigation of the dynamic properties of the signal received from the sensitive element, taking into account the interaction of various characteristic quantities, requires the development of an improved mathematical apparatus due to the difficulties arising during the primary and secondary signal conversion by parts of the sensor, signal transmission elements.

The analytical expression for the output voltage study takes into account the location of the current-carrying coils of multiphase primary currents in the sensor structure, the shape of the magnetic transducer and the type of sensitive part, the number of windings and the corresponding parameters, is formed as follows [5-12]:

$$
\begin{aligned}
& U_{E a}(t)=-R_{E c e l} I_{E a}(t)-\left(L_{E c e l} d I_{E a}(t)\right) / d t+\left(W_{2} d \Phi_{B}(t)\right) / d t+\left(W_{3} d \Phi_{C}(t)\right) / d t \\
& U_{E b}(t)=-R_{E c e l} I_{E b}(t)-\left(L_{E c e 2} d I_{E b}(t)\right) / d t+\left(W_{c e 2} d \Phi_{A}(t)\right) / d t+\left(W_{E 3} d \Phi_{C}(t)\right) / d t \\
& \left.U_{E c}(t)=-R_{E c e l} I_{E c}(t)-\left(L_{E c e 3} d I_{E C}(t)\right) / d t+\left(W_{1} d \Phi_{A}(t)\right) / d t+\left(W_{3} d \Phi_{B}(t)\right) / d t\right)
\end{aligned}
$$

where $R_{E c e l}, L_{E c e l}, R_{E c e l}, L_{E c e 2}, W_{c e 2}, W_{E 3}, R_{E c e l}, L_{E c e 3}$ - are active resistance, inductance of the sensitive part, mutual inductance between the primary winding and the sensitive winding, $W_{1}, W_{2}, W_{3}$ - define the number of the sensitive part windings,

$I_{E a}, I_{E \sigma}, I_{E c}-$ are the secondary output currents to sensitive parts. 
If the inductance of electrical networks of reactive power sources PS be equal to : $\mathrm{L}_{\mathrm{EI}}=$ $\mathrm{L}_{\mathrm{EII}}=\mathrm{L}_{\mathrm{EIII}}=0$; there is an increase in the initial value of multiphase currents in the networks, then the magnetic currents gradually reach a stable sinusoidal value in the switching part [3-6].

Installed rated reactive power of cosine capacitors can be widely used as reactive power sources PS, is $\mathrm{Q}_{\mathrm{N} \mathrm{KKU}}=50 \mathrm{kVA}$. $\mathrm{I}_{\mathrm{Ek} \mathrm{A}}=\mathrm{I}_{\mathrm{Ek} \mathrm{B}}=\mathrm{I}_{\mathrm{Ek} \mathrm{C}}=131$ Ampere.

Installed rated reactive power $\mathrm{Q}_{\mathrm{N} \mathrm{KKU}}=50 \mathrm{kVA}$ capacitor voltage $\mathrm{U}_{\mathrm{EkA}}=\mathrm{U}_{\mathrm{EkB}}=\mathrm{U}_{\mathrm{EkC}}=380$ $\mathrm{V}$, if the capacitors are connected in a triangular circuit, the currents entering the sensor flowing from the network will be $\mathrm{I}_{\mathrm{Ek} \mathrm{A}}=\mathrm{I}_{\mathrm{Ek} \mathrm{B}}=\mathrm{I}_{\mathrm{EkC}}=76 \mathrm{~A}$. In this case, the inductance of the network connected to the reactive power sources is $\mathrm{L}_{\mathrm{EI}}=\mathrm{L}_{\mathrm{EII}}=\mathrm{L}_{\mathrm{EIII}}=10-3 \mathrm{H}$, capacity $\mathrm{S}_{\mathrm{EA}}=\mathrm{S}_{\mathrm{EB}}=\mathrm{S}_{\mathrm{EC}}=10-7 \mathrm{~F}$, impedance $\mathrm{Z}_{\mathrm{EA}}=\mathrm{Z}_{\mathrm{EB}}=\mathrm{Z}_{\mathrm{EC}}=0,289 \Omega$. The maximum values of magnetic fluxes are determined as follows:

$$
F_{\max A}=F_{\max B}=F_{\max C}=U_{\max } /\left(W_{I \omega}\right)=380 /(1 \cdot 2 \cdot 3,14 \cdot 50)=0,70637 \mathrm{~Wb} .
$$

and $E_{a g}, U_{a \gamma}, U_{B \gamma}, U_{c \gamma}, U_{a \Delta}, U_{в \Delta}$ and $U_{c \Delta}$ - are the secondary signal supply signal for monitoring and control of primary currents flowing from reactive power sources and PS networks. The dynamic characteristics of the sensor are investigated using the following analytical expression, generated based on the graph model shown in Figure 3. [6,7-12]:

$$
\begin{gathered}
U_{A \gamma}=K_{\Phi \mu U e} \Pi_{\mu 1} W\left(F_{\mu 11}, F_{\mu 14}\right) K_{\text {IeF } \mu} I_{A \gamma} \sin (\omega t)+I_{A m} e^{\wedge}(-t / T) \\
U_{B \gamma}=K_{\Phi \mu U e} \Pi_{\mu 2} W\left(F_{\mu 21}, F_{\mu 24}\right) K I_{e F \mu} I_{B \gamma}\left(\sin \omega t+120^{\circ}\right)+I_{B m} e^{\wedge}(-t / T) \\
U_{C \gamma}=K_{\Phi \mu U e} \Pi_{\mu 3} W\left(F_{\mu 31}, F_{\mu 34}\right) K I_{e F \mu} I_{C \gamma}\left(\sin \omega t-120^{\circ}\right)+I_{C m} e^{\wedge}(-t / T) \\
U_{A \Delta}=K_{\Phi \mu U e} \Pi_{\mu 4} W\left(F_{\mu 41}, F_{\mu 44}\right) K_{I e F \mu} I_{A \Delta} \sin (\omega t)+I_{A m} e^{\wedge}(-t / T) \\
U_{B \Delta}=K_{\Phi \mu U e} \Pi_{\mu 5} W\left(F_{\mu 51}, F_{\mu 54}\right) K_{I e F \mu} I_{B \Delta}\left(\sin \omega t+120^{\circ}\right)+I_{B m} e^{\wedge}(-t / T) \\
U_{C \Delta}=K_{\Phi \mu U e} \Pi_{\mu 6} W\left(F_{\mu 61}, F_{\mu 64}\right) K_{I e F \mu} I_{C \Delta}\left(\sin \omega t-120^{\circ}\right)+I_{C m} e^{\wedge}(-t / T)
\end{gathered}
$$

where: $\Pi_{\mu j}=\left(\mu_{0} F_{j}\right) / \delta_{\mu j}(\mathrm{j}=1 . .6)$ defines a magnetic parameter of the variable part of the generated sensor $U_{\text {output }}$ output output voltages $\left(\mu_{0^{-}}\right.$magnetic absorption of air gaps with sensing element, $\left.\mu_{0}=1,2510^{-6} \mathrm{H} / \mathrm{m}\right)$;

$\mathrm{S}-$ is the cross-sectional area of the magnetic circuit section on which the sensitive elements are installed, for example $\mathrm{a}_{\mathrm{xb}}=0,01 \cdot 0,01 \mathrm{~m}^{2}$;

$\delta_{\mu}-$ is an air gap heights with sensing elements (m);

$W\left(F_{\mu i j}, F_{\mu i n}\right)$ is a transfer function of the magnetic switching part.

\section{Results and Discussion}

The primary input currents of dynamic changes occurring in the sensor, depending on the magnetic currents' values and parameters and the output voltages they generate, are shown in the graphs of changes (Fig. 4) and (Fig. 5). 


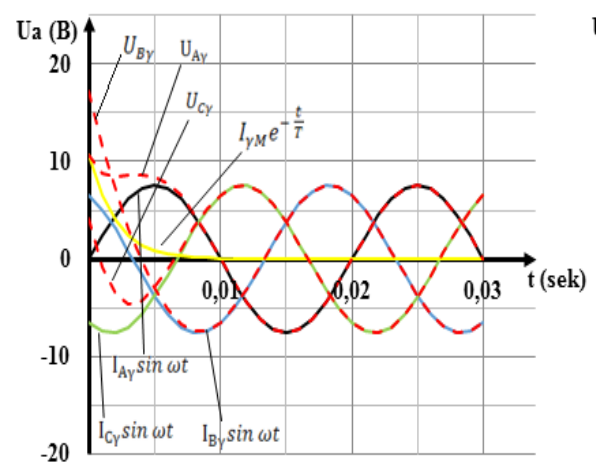

a)

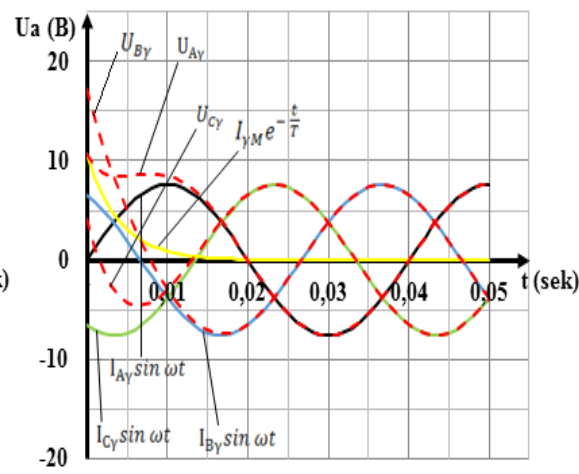

b)

Fig. 4 Dynamic characteristics of the sensor primary currents' dependence on the output voltage (sources of PS reactive power are connected in a star model: a) at $\mathrm{T}=0,02 \mathrm{~b}$ ) at $\mathrm{T}=0,04$ ).

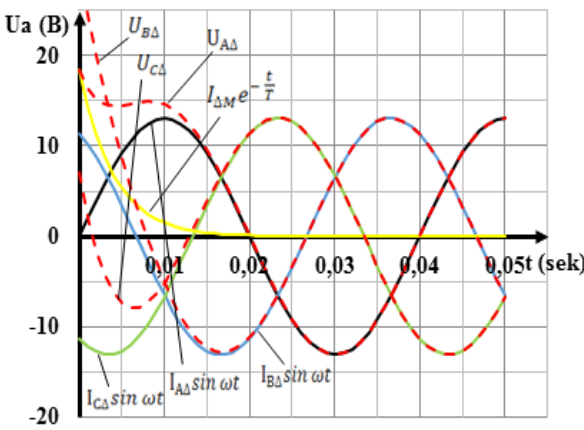

a)

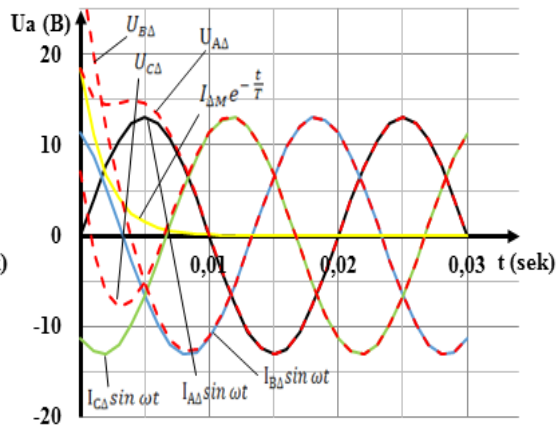

b)

Fig. 5. Dynamic characteristics of the relationship between the primary currents and secondary voltages of the sensor (sources of reactive power PSS connected by a triangle: $a$ ) at $T=0,02 \mathrm{~b}$ ) at $\mathrm{T}=$ $0,04)$.

Depending on the primary input currents, dynamic changes occurring in the sensor, the values and parameters of the magnetic currents and the output voltages that they generate, from the graphs of changes (Fig. 4) and (Fig. 5), it can be concluded, that the currents flow from the primary coils of reactive power sources. In case of 0.02 seconds, $0.008-0.012$ seconds after connecting to PS networks with RES, $t=0,04$ seconds and 0,015 - 0,025 seconds after connecting to the networks PS with RES, output voltage values are stabilized.

\section{Conclusion}

Analysis of devices and methods for regulating reactive power in PS with RES showed that research on the generation and management of reactive power is necessary to ensure an uninterrupted and high-quality supply of electricity to consumers.

The features of sensor signals for monitoring and control of reactive power sources in PS RES, which are an important tool for ensuring mutual power synchronization, are based on ensuring sufficient reactive power generation for PS voltage regulation. It has been found that this gives an opportunity for uninterrupted connection of energy and power to PS with RES.

Analysis of signal conversion processes occurring in electromagnetic sensors and 
structural foundations of sensor elements for multiphase current control PS RES and control of reactive power sources has shown that they meet basic requirements such as the conversion of multiphase primary currents into a secondary voltage signal and speed.

The magnetic currents generated by PS power supplies and generated by the currents $I_{A \gamma}, I_{B \gamma}, I_{C \gamma}, I_{A \Delta}, I_{B \Delta}$ and $I_{C \Delta}$, flowing through electrical networks are the primary currents in magnetic circuits $F_{\mu}-$ m.yu.k. A graph model to study the conversion processes into secondary output voltages $\mathrm{U}_{\text {output }}$ has been created.

When studying dynamic changes occurring in an electromagnetic sensor, depending on the magnitude of input currents, magnetic fluxes and output voltages, the inertia of the loads is $\mathrm{T}=0,02 \mathrm{~s}$, when the sensor is connected to the network at the time $0,008-0,012 \mathrm{~s}$. As a result of the observations, it was found that it reaches its steady state in the time interval $0.015-0.025 \mathrm{~s}$ at $\mathrm{t}=0,04 \mathrm{~s}$, moreover, these indicators prove that the sensor is capable of generating a secondary signal at high speed.

\section{References}

1. I.Kh. Siddikov, M.A. Anarbaev, A.A. Abdumalikov, A.B. Abubakirov, M.T. Maxsudov, I.M. Xonturaev, International Conference on Information Science and Communications Technologies Applications, Trends And Opportunities, 1-6 (2019)

2. I.Kh. Siddikov, A.B. Abubakirov, A.J. Allanazarova, R.M. Tanatarov, Sh.B. Kuatova, Solid State Technology 63 (6), 13143-13148 (2020)

3. A.B. Abubakirov, A.A. Yo'ldashev, I.Q. Baymuratov, M.T. Sharipov, A.D. Utemisov, EPRA International Journal of Research and Development, India 5, 214-218 (2020)

4. I. Siddikov, Kh. Sattarov, A.B. Abubakirov, M. Anarbaev, I. Khonturaev, M. Maxsudov, Research of transforming circuits of electromagnets sensor with distributed parameters, 10 th International Symposium on intelegent Manufacturing and Service Systems. 9-11 September 2019. Sakarya. Turkey, 831-837 (2019)

5. A.B. Abubakirov, European science review, Scientific journal, Vienna, Austria 5(6), 269-273 (2018)

6. I.Kh. Siddikov, A.B. Abubakirov, A.A. Yo'ldashov, G.Z. Babaxova, I.M. Xontoraev, N.N. Mirzoev, European science review, Scientific journal, Vienna, Austria 1(2), 248251 (2018)

7. I.Kh. Siddikov, Kh.A. Sattarov, A.B. Abubakirov, A.Zh. Esenbekov, D.B. Sarsenbaev, Bulletin of Karakalpak Scientific Research Institute of Natural Sciences, Nukus 2, 2732 (2018)

8. I.Kh. Siddikov, A.B. Abubakirov, K.M. Nazhmatdinov, A.Zh. Esenbekov, Bulletin of Karakalpak Scientific Research Institute of Natural Sciences. Nukus 2, 66-68 (2017)

9. I.Kh. Siddikov, A.B. Abubakirov, K.M. Nazhmatdinov, A.Zh. Esenbekov, Bulletin of Karakalpak Scientific Research Institute of Natural Sciences. Nukus 2, 66-68 (2017)

10. V.G. Lukashkin, V.K. Garipov, V.V. Sleptsov, A.V. Vishnekov, Automation of measurements, control and management (Mechanical Engineering, Moscow, 2005)

11. H. Schaumburg, Werkstoffe und Bauelemente der Elektrotechnik. Sensoren (Stuttgart: B.G. Teubner, 1992)

12. M. Dominic, M. Yiannos, CMOS Circuits for Electromagnetic Vibration Transducers: Interfaces for Ultra-Low Voltage Energy Harvesting, (2015) 